\title{
Fluorescence in Erbium Doped Gallium Lanthanum Sulphide: Potential for mid-IR Waveguide Laser
}

\author{
Giorgos Demetriou, ${ }^{1 *}$ Fiona Thorburn, ${ }^{1}$ Adam Lancaster, ${ }^{1}$ Chris Craig, ${ }^{2}$ Ed Weatherby, ${ }^{2}$ Daniel W \\ Hewak, ${ }^{2}$ and Ajoy.K Kar ${ }^{1}$ \\ ${ }^{1}$ Institute of Photonics and Quantum Sciences, School of Engineering and Physical Sciences, David Brewster Building, Heriot- \\ WattUniversity, Edinburgh, EH11 4AS, United Kingdom \\ ${ }^{2}$ Optoelectronics Research Centre, University of Southampton, Southampton SO17 1BJ \\ *gd123@hw.ac.uk
}

\begin{abstract}
Fluorescence is reported in waveguides fabricated via the ultrafast laser inscription technique in Erbium doped Gallium Lanthanum Sulphide $\left(\mathrm{Er}^{3+}: \mathrm{GLS}\right)$ for mid-infrared laser applications. The pump wavelength was $980 \mathrm{~nm}$ leading to mid-infrared transition at $2.75 \mu \mathrm{m}$.
\end{abstract}

\section{Introduction.}

Integrated optics that operate in the mid-IR region of the electromagnetic spectrum $(3-25 \mu \mathrm{m})$ are attracting a considerable amount of research interest due to their potential applications such as remote gas sensors[1] and stellar interferometry[2]. Materials such as chalcogenide glasses are suitable host materials for such devices due to their excellent mid-IR transparency[3] which extends beyond $8 \mu \mathrm{m}$.

Chalcogenide glasses are based on the chalcogen elements S, Se, and Te[4]. These glasses are formed by the addition of other elements such as Ge, As, Sb, Ga, etc. In addition to their transparency in the mid-IR they also may also offer a high nonlinear refractive index up to $~ 500$ times that of fused silica[5], low-multi-photon absorption and high photosensitivity. Of the chalcogenide glasses, gallium lanthanum sulphide (GLS) is a particularly appealing candidate as it is thermally stable up to $550^{\circ} \mathrm{C}$, arsenic free and non-hydroscopic.

These glasses have several advantages over other glass materials as rare-earth hosts. The solubility of rareearth ions is extremely high due to the presence of lanthanum as a glass former, and the emission cross sections of rare-earth levels are enhanced by the high refractive index $(\mathrm{n}=2.4$ at $1.55 \mu \mathrm{m})$. Combined with the low phonon energy, this potentially gives access to MIR transitions for lasers.

The non-toxicity, high glass transition temperature, excellent rare-earth solubility, high refractive index $(\mathrm{n}=$ 2.3 at $1 \mu \mathrm{m}$ ), low phonon-energy, high non-linearity and photosensitive properties of GLS also make it an interesting candidate for research into planar waveguide devices[6].

\section{Waveguide fabrication.}

GLS glass fabrication is described in detail in reference[7]. In our experiments, GLS glass with a composition of $65 \mathrm{~mol} \%$ gallium sulphide and $35 \mathrm{~mol} \%$ lanthanum sulphide were melted and after annealed polished into flats $10 \times 10 \times 1 \mathrm{~mm}$ in size with all six faces polished to $\lambda / 4$. In this work we utilized the ultrafast laser inscription (ULI) technique[8] to fabricate waveguides embedded in an $\mathrm{Er}^{3+}$ :GLS substrate. ULI is a powerful fabrication technique which relies on the nonlinear absorption of sub-bandgap photons to induce permanent structural changes to a material. These changes can manifest themselves in multiple ways including a change in refractive index[8]. The induced modification can be localized to the high intensity region at the focus of an ultra-short pulse train. This gives ULI the unique advantage over other waveguide fabrication techniques of being capable of forming three dimensional structures[8].

The waveguides were fabricated using a mode-locked Yb-doped fiber laser which emitted 400 fs pulses with a central wavelength of $1060 \mathrm{~nm}$ and a pulse repetition rate of $500 \mathrm{kHz}$.

The substrate was mounted on air bearing stages and pulses from the fabrication laser were focused inside the substrate to a distance of $360 \mu \mathrm{m}$ from the top surface using $0.4 \mathrm{NA}$ aspheric lens. The multi-scan writing technique was used. Fabrication pulse energies incident onto the sample were varied from 60-40 nJ in 
decreasing increments of $4 \mathrm{~nJ}$. The translation speeds varied from 0.5 to $20 \mathrm{mms}^{-1}$ with the substrate translation being perpendicular to the laser beam direction.

\section{Waveguide Characterisation}

$\mathrm{The}{ }^{3+}$ ions in the waveguides were excited at the energy level ${ }^{4} \mathrm{I}_{11 / 2}$ using a fibre laser operating at $980 \mathrm{~nm}$ which was collimated with a 10X aspheric lens and then focussed in the sample with another 10X aspheric lens. The fluorescence was imaged over a distance of $83 \mathrm{~cm}$ with a $\mathrm{CaF}_{2}$ lens and captured using a FLIR SC7000 camera using a long pass filter which cut off at $2.0 \mu \mathrm{m}$ in front of the camera in order to block the pump wavelength and the $1550 \mathrm{~nm}$ wavelength from the ${ }^{4} \mathrm{I}_{13 / 2} \rightarrow{ }^{4} \mathrm{I}_{15 / 2}$ transition. Waveguides of heights and widths up to $20 \times 12 \mu \mathrm{m}$ are shown to exhibit single mode guiding at $2.75 \mu \mathrm{m}$ with very symmetric mode profiles. Figure 1 shows the facet image of one of the waveguides along with the corresponding mode profile image. In addition the absorption spectrum of $9.7 \mathrm{~mol} \% \mathrm{Er}^{3+}$ doped GLS glass and the $\mathrm{Er}^{3+}$ energy levels indicating the infrared transitions are also presented in the same figure.
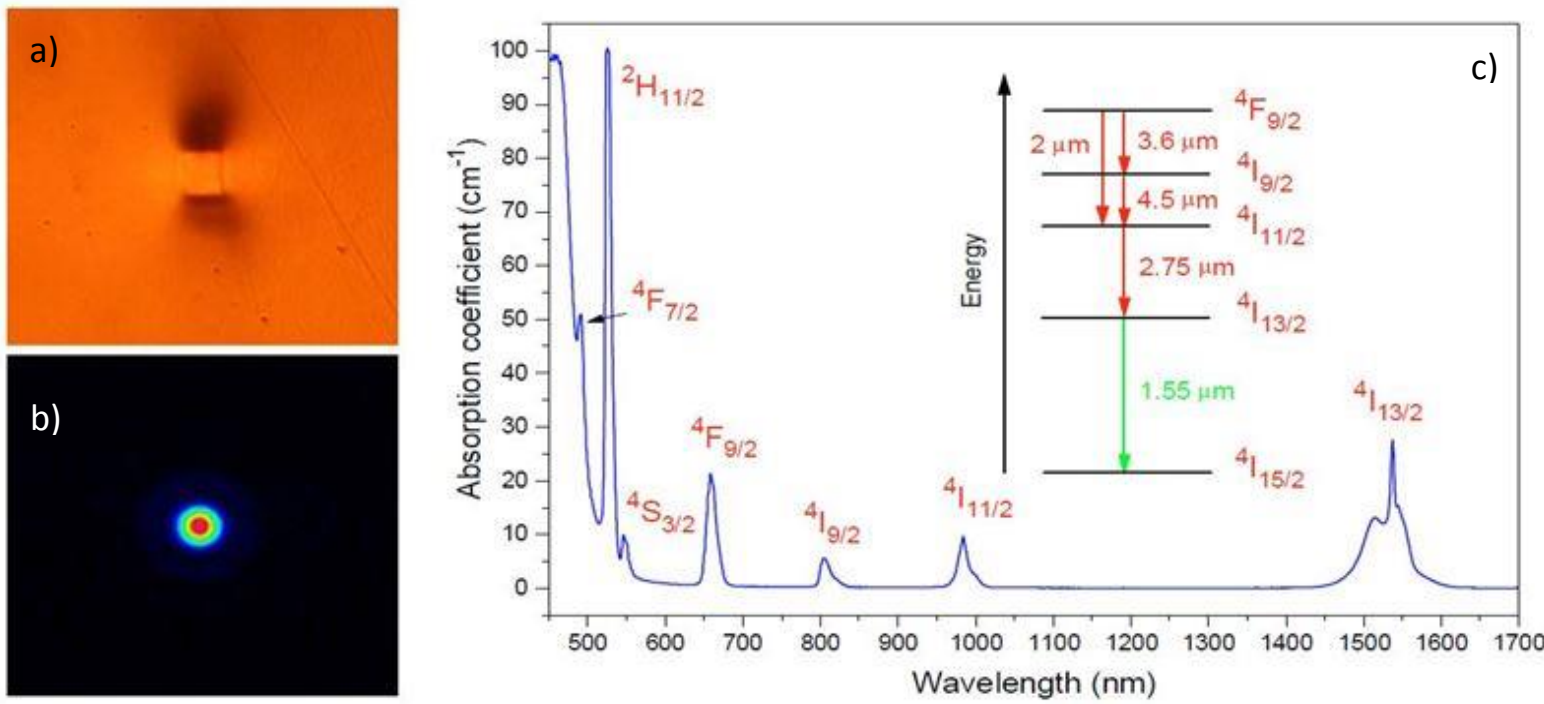

Fig 1. (a) Facet image for a waveguide taken in transmission mode, (b) Corresponding mode profile image of fluorescence at $2.75 \mu \mathrm{m}$, (c) Absorption spectrum of $9.7 \mathrm{~mol} \% \mathrm{Er}^{3+}$ doped GLS glass and $\mathrm{Er}^{3+}$ energy levels indicating the infrared transitions

\section{Conclusion}

In conclusion we present the fabrication of waveguides in $\mathrm{Er}^{3+}$ doped gallium lanthanum sulphide by utilizing the ULI technique. We demonstrated fluorescence at $2.75 \mu \mathrm{m}$ with a pump wavelength of $980 \mathrm{~nm}$ which enables us to explore the possibilities for an $\mathrm{Er}^{3+}$ :GLS waveguide laser

\section{References}

1. A. Kosterev, G. Wysocki, Y. Bakhirkin, S. So, R. Lewicki, M. Fraser, F. Tittel, and R. F. Curl, "Application of quantum cascade lasers to trace gas analysis," Appl. Phys. B 90, 165-176 (2008).

2. P. Houizot, C. Boussard-Plédel, A. J. Faber, L. K. Cheng, B. Bureau, P. A. Van Nijnatten, W. L. M. Gielesen, J. Pereira do Carmo, and J. Lucas, "Infrared single mode chalcogenide glass fiber for space," Opt. Express 15, 12529-12538 (2007).

3. B. J. Eggleton, B. Luther-Davies, and K. Richardson, "Chalcogenide photonics," Nat Photon 5, 141-148 (2011)

4. A. Zakery and S. R. Elliott, "Optical properties and applications of chalcogenide glasses: a review," Journal of Non-Crystalline Solids 330, 1-12 (2003).

5. K. S. Bindra, H. T. Bookey, A. K. Kar, B. S. Wherrett, X. Liu, and A. Jha, "Nonlinear optical properties of chalcogenide glas ses: Observation of multiphoton absorption," Applied Physics Letters 79, 1939-1941 (2001).

6. $\quad$ D. W. Hewak, "The promise of chalcogenides," Nat Photon 5, 474-474 (2011).

7. Y. D. West, T. Schweizer, D. J. Brady, and D. W. Hewak, "Gallium Lanthanum Sulphide Fibers for Infrared Transmission," Fiber and Integrated Optics 19, 229-250 (2000).

8. D. Choudhury, J. R. Macdonald, and A. K. Kar, "Ultrafast laser inscription: perspectives on future integrated applications," Laser \& Photonics Reviews 8, 827-846 (2014). 